\title{
Sur une opérade ternaire liée aux treillis de Tamari
}

\author{
F. Chapoton
}

12 octobre 2018

\begin{abstract}
Résumé
On introduit une opérade anticyclique $\mathbf{V}$ définie par une présentation ternaire quadratique. On montre qu'elle admet une base indexée par les arbres binaires plans. On relie cette construction à la famille des treillis de Tamari $\left(\mathrm{Y}_{n}\right)_{n \geq 0}$ en construisant un isomorphisme entre $\mathbf{V}(2 n+1)$ et le groupe de Grothendieck de la catégorie $\bmod Y_{n}$ qui envoie la base de $\mathbf{V}(2 n+1)$ sur les classes des modules projectifs et qui transforme la structure anticyclique de $\mathbf{V}$ en la transformation de Coxeter de la catégorie dérivée de $\bmod \mathrm{Y}_{n}$. La dualité de Koszul des opérades permet alors de calculer le polynôme caractéristique de cette transformation de Coxeter en utilisant une transformation de Legendre.
\end{abstract}

\begin{abstract}
We introduce an anticyclic operad $\mathbf{V}$ given by a ternary generator and a quadratic relation. We show that it admits a natural basis indexed by planar binary trees. We then relate this construction to the familly of Tamari lattices $\left(\mathrm{Y}_{n}\right)_{n \geq 0}$ by defining an isomorphism between $\mathbf{V}(2 n+1)$ and the Grothendieck group of the category $\bmod Y_{n}$. This isomorphism maps the basis of $\mathbf{V}(2 n+1)$ to the classes of projective modules and sends the anticyclic map of the operad $\mathbf{V}(2 n+1)$ to the Coxeter transformation of the derived category of $\bmod Y_{n}$. The Koszul duality theory for operads then allows to compute the characteristic polynomial of the Coxeter transformation by a Legendre transform.
\end{abstract}

Keywords: operad ; Dendriform operad ; anticyclic operad ; ternary operad ; binary tree ; Tamari lattice ; Coxeter transformation

MSC2010: 18D50；05C05；06A11

\section{$0 \quad$ Introduction}

Les arbres binaires plans sont des objets combinatoires très classiques, qui sont apparus depuis quelques années dans des situations algébriques variées, dont l'une des plus remarquables est la description des algèbres dendriformes libres à l'aide d'arbres binaires plans, due à J.-L. Loday [23. Ce résultat s'exprime, dans la cadre conceptuel des opérades, comme la description de l'opérade Dendriforme en termes d'arbres binaires plans. 
Dans l'étude des algèbres dendriformes libres et de l'opérade Dendriforme, poursuivie depuis par différents auteurs 7, 8, 16, 31, 1, 24, il est progressivement devenu clair qu'une famille de posets jouait un rôle fondamental. Ce sont les treillis de Tamari, initialement introduits par D. Tamari de façon purement combinatoire [32] en termes de parenthèsages. Ces treillis apparaissent en théorie des représentations de deux façons distinctes, soit comme ordres partiels sur les modules basculants 30, 15, soit parmi les treillis cambriens, comme ordres partiels sur les amas, dans la théorie des algèbres amassées de S. Fomin et A. Zelevinsky [9, 29].

Un aspect intriguant de cette relation profonde entre l'opérade dendriforme et les treillis de Tamari est le point suivant. Si $Y_{n}$ est l'ensemble des arbres binaires plans à $n$ sommets, on peut définir deux applications linéaires de $\mathbb{Z} Y_{n}$ dans lui-même. La première application $\tau$ provient de la structure anticyclique de l'opérade Dendriforme; elle est en particulier périodique, de période $n+1$. La seconde application $\theta$ provient de la catégorie $\bmod \mathrm{Y}_{n}$ des modules sur le poset $Y_{n}$. Elle décrit l'action d'un endofoncteur naturel de la catégorie dérivée $D \bmod Y_{n}$ de cette catégorie. Il se trouve que l'application $\tau$ est (au signe près) le carré de $\theta$ 2. En particulier, l'application $\theta$ est périodique, de période $2 n+2$.

Dans un article précédent [4, cette situation a été décrite et précisée en introduisant une catégorification de l'opérade Dendriforme via les catégories de modules sur les treillis de Tamari. Cette construction utilise de manière essentielle une famille d'éléments de l'opérade Dendriforme indexée par les arbres noncroisés. Il se trouve que les arbres non-croisés sont en bijection avec les arbres ternaires, qui forment une base de l'opérade ternaire libre sur un générateur. On peut donc se demander si une opérade ternaire ne pourrait pas jouer un rôle dans le contexte dendriforme.

L'objet du présent article est précisément de présenter une relation entre les treillis de Tamari et une certaine opérade ternaire. Cette opérade $\mathbf{V}$ est engendrée par un élément impair de degré 3 modulo une unique relation quadratique. On montre qu'elle admet en degré $2 n+1$ une base indexée par les arbres binaires plans à $n$ sommets, en utilisant la méthode des bases de Gröbner pour les opérades [17, 6.

On montre que l'opérade $\mathbf{V}$ possède une structure anticyclique, ce qui donne une application linéaire $\theta_{\mathbf{V}}$ de période $2 n+2$ sur le groupe abélien $\mathbf{V}(2 n+1)$. Le premier résultat principal de l'article est un isomorphisme entre $\mathbf{V}(2 n+1)$ et $\mathbb{Z} Y_{n}$ qui identifie $\theta_{\mathbf{V}}$ et $\theta$ et donne donc une nouvelle preuve de la périodicité de $\theta$ mentionnée plus haut.

On utilise cette description de $\theta$ pour en obtenir le polynôme caractéristique, pour lequel une conjecture a été formulée dans 3. On utilise pour cela une fonction symétrique associée à chaque opérade cyclique, qui sert à coder l'action du groupe cyclique sur les composantes de cette opérade. Il se trouve que les fonctions symétriques associées à deux opérades cycliques duales de Koszul sont reliées par une transformation de Legendre. Il suffit donc de calculer la fonction symétrique associée à la duale de Koszul de $\mathbf{V}$, qui se trouve être très simple, puis sa transformée de Legendre. Cette même technique a déjà été employée pour calculer le polynôme caractéristique de $\tau$ en utilisant sa relation avec l'opérade Dendriforme.

On dispose donc ainsi de deux opérades dont la structure anticyclique est fortement reliée aux treillis de Tamari, chacune à sa manière. L'opérade Dendriforme permet de décrire le carré de $\theta$, et la base la plus naturelle de cette 
opérade correspond aux modules simples sur les treillis de Tamari. L'opérade V permet de décrire $\theta$ et possède une base correspondant aux modules projectifs. Ces deux structures forment ensemble une structure algébrique assez complexe et remarquable.

Pour terminer cette introduction, voici quelques mots sur le contexte en théorie des représentations. La périodicité de la transformation de Coxeter pour la catégorie $D \bmod Y_{n}$ se place en fait dans un ensemble plus vaste de conjectures. Le treillis de Tamari $Y_{n}$ peut en effet être considéré soit comme le treillis cambrien associé aux carquois de type $A_{n}$ équiorienté, soit comme le poset des modules basculants associé aux carquois de type $A_{n+1}$ équiorienté. La propriété de périodicité semble également vraie pour tous les treillis cambriens et pour tous les posets de modules basculants associés aux carquois de type $A D E$.

Pour l'instant, cette propriété est démontrée pour tous les carquois de type $A$. Elle résulte du cas équiorienté, démontré ici ou dans 2 en utilisant la théorie des opérades, et des théorèmes de Ladkani montrant l'équivalence dérivée entre les treillis cambriens et les posets de modules basculants 20, 19 lorsque le carquois change par mutation en un puits ou une source.

Cette périodicité n'est par ailleurs qu'une conséquence d'une propriété conjecturale plus forte. En effet, elle est vraie si les catégories dérivées $D \bmod Y_{n}$ sont Calabi-Yau fractionnaires. Pour l'instant, on sait seulement que cette propriété plus forte est vraie pour $n \leq 3$, où les catégories $D \bmod Y_{n}$ admettent une description simple en termes de catégories de modules sur les carquois $A_{1}, A_{2}$ et $D_{5}$.

\section{Construction et propriétés de $\mathrm{V}$}

\subsection{Généralités et notations}

On se place dans la catégorie monoïdale symétrique des groupes abéliens gradués, avec la règle des signes de Koszul par rapport à cette graduation. Les morphismes sont les applications linéaires respectant la graduation.

Pour le cadre général de la théorie des opérades, on renvoie le lecteur aux ouvrages [28, 33 .

On ne travaille dans cet article qu'avec des opérades non-symétriques, qu'on appelle simplement des opérades.

Soit $\mathbf{P}$ une collection de groupes abéliens gradués $\mathbf{P}(n)=\oplus_{k \in \mathbb{Z}} \mathbf{P}(n)_{k}$ pour $n \geq 1$ et soit $a \in \mathbf{P}(n)_{k}$. On appelle $n$ le degré de $a$, noté \#a. On appelle $k$ le poids de $a$, simplement noté $a$ dans les exposants de -1 , par un abus de notation commode qui ne porte pas à confusion. La règle des signes de Koszul s'applique uniquement à la graduation par le poids.

On rappelle brièvement la définition des opérades.

Définition 1 Une opérade $\mathbf{P}$ est la donnée d'une collection de groupes abéliens gradués $\mathbf{P}(n)=\oplus_{k \in \mathbb{Z}} \mathbf{P}(n)_{k}$ pour $n \geq 1$, d'une unité $\mathbf{1} \in \mathbf{P}(1)_{0}$ et d'applications linéaires

$$
\circ_{i}: \mathbf{P}(m) \otimes \mathbf{P}(n) \longrightarrow \mathbf{P}(m+n-1)
$$

pour $1 \leq i \leq m$. Les compositions $\circ_{i}$ doivent vérifier

$$
\left(a \circ_{i} b\right) \circ_{j+i-1} c=a \circ_{i}\left(b \circ_{j} c\right),
$$


et

$$
\left(a \circ_{i} b\right) \circ_{j+\# b-1} c=(-1)^{b c}\left(a \circ_{j} c\right) \circ_{i} b \quad \text { si } \quad i<j .
$$

On note $\circ_{\max }$ pour la composition la plus à droite, i.e.

$$
a \circ_{\max } b=a \circ_{\# a} b .
$$

On rappelle aussi la définition des opérades anticycliques.

Définition 2 Une structure d'opérade anticyclique sur une opérade $\mathbf{P}$ est la donnée pour tout $n \geq 1$ d'un opérateur $\theta$ sur $\mathbf{P}(n)$ vérifiant $\theta(\mathbf{1})=-\mathbf{1}$, $\theta^{n+1}=\mathrm{Id}$ et tels qu'on ait les axiomes suivants :

$$
\theta\left(a \circ_{i} b\right)=\theta(a) \circ_{i-1} b \quad \text { si } \quad i>1,
$$

et

$$
\theta\left(a \circ_{1} b\right)=-(-1)^{a b} \theta(b) \circ_{\max } \theta(a) .
$$

Remarque 1.1 La notion opérade cyclique est définie similairement, mais avec deux changements de signes : $\theta(\mathbf{1})=\mathbf{1}$ et le coté droit de (6) est remplacé par son opposé.

\subsection{L'opérade anticyclique ternaire $\mathrm{V}$}

Soit V l'opérade définie par la présentation par générateurs et relations suivante.

On se donne un générateur $\square$ de degré 3 et de poids 1 et on impose la relation

$$
\square \circ_{1} \square-\square \circ_{2} \square+\square \circ_{3} \square=0 .
$$

On introduit sur $\mathbf{V}$ une structure anticyclique.

Proposition 1.2 Il existe sur $\mathbf{V}$ une unique structure d'opérade anticyclique, donnée pour tout $n \geq 0$ par un opérateur $\theta_{\mathbf{V}}$ sur $\mathbf{V}(2 n+1)$, telle que

$$
\theta_{\mathbf{V}}(\square)=-\square
$$

Preuve. Par la théorie générale des opérades anticycliques, il suffit de vérifier la compatibilité de $\theta_{\mathbf{V}}$ avec la relation (7), en montrant que l'image par $\theta_{\mathbf{V}}$ de cette relation est un multiple de cette relation. On obtient, en utilisant (5) et (6) et en gardant les termes dans l'ordre initial,

$$
\square \circ_{3} \square+\square \circ_{1} \square-\square \circ_{2} \square,
$$

qui est bien proportionnel à (7).

\subsection{Base $Q$ de V}

On donne ici une base explicite de $\mathbf{V}$.

Définition 3 Un arbre binaire plan est un graphe connexe et simplement connexe, muni d'un plongement dans le plan considéré à isotopie près, dont les sommets sont soit trivalents (sommets internes) soit univalents, et muni d'un sommet univalent distingué (racine). On appelle feuilles les sommets univalents non distingués. 


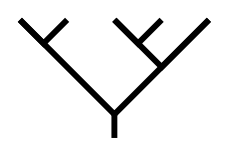

FiguRE 1 - Un arbre binaire plan dans $\mathrm{Y}_{4}$

On convient de dessiner les arbres binaires plans avec leurs feuilles en haut et leur racine en bas. Par commodité, on oriente (implicitement) les arêtes en direction de la racine.

Un arbre binaire plan est soit l'arbre trivial | sans sommet interne, soit se décompose de manière unique, par enlèvement de l'arête entrante en sa racine, en une paire $(x, y)$ d'arbres binaires plans.

Pour $n \geq 0$, on note $\mathrm{Y}_{n}$ l'ensemble des arbres binaires plans à $n$ sommets internes.

A chaque élément $x$ de $\mathrm{Y}_{n}$, on associe un élément $Q_{x}$ de $V(2 n+1)_{n}$ par récurrence $\operatorname{sur} n$. Si | est l'unique arbre sans sommet, on pose $Q_{\mid}=\mathbf{1}$, l'unité de l'opérade $\mathbf{V}$. Sinon, on associe à l'arbre binaire plan $z$ qui se décompose en une paire $(x, y)$ l'élément

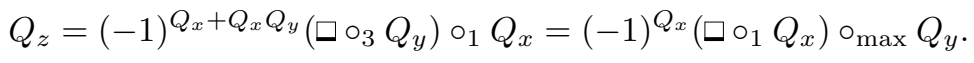

L'égalité de ces deux expressions résulte de l'axiome (3) des opérades.

Par exemple, on a $Q_{\curlyvee}=\square$ et on associe à l'arbre $x$ de la figure 11'élément

$$
Q_{x}=\left(\square \circ_{3}\left(\square \circ_{1} \square\right)\right) \circ_{1} \square .
$$

On note $Q$ l'ensemble des éléments $Q_{x}$ ainsi définis.

On utilise ci-dessous le formalisme des bases de Gröbner pour les opérades, voir [17, 6] et [33, §8.1].

Proposition 1.3 La relation (17) forme une base de Gröbner de l'idéal qu'elle engendre dans l'opérade libre sur $\square$, pour un ordre admissible dans lequel la composition $\mathrm{O}_{2}$ domine les compositions $\mathrm{o}_{1}$ et $\mathrm{o}_{3}$.

Preuve. Pour cela, il suffit de montrer une propriété de confluence pour la réécriture de la paire critique

$$
\left(\square \circ_{2} \square\right) \circ_{3} \square=\square \circ_{2}\left(\square \circ_{2} \square\right) \text {. }
$$

Il s'agit de vérifier que les deux calculs obtenus en appliquant à volonté

$$
\square \circ_{2} \square \longrightarrow \square \circ_{1} \square+\square \circ_{3} \square
$$

à cette expression donnent le même résultat.

D’une part, la réécriture en partant de $\left(\square \circ_{2} \square\right) \circ_{3} \square$ donne

$$
\left(\square \circ_{1} \square+\square \circ_{3} \square\right) \circ_{3} \square=\square \circ_{1}\left(\square \circ_{3} \square\right)+\square \circ_{3}\left(\square \circ_{1} \square\right) \text {. }
$$

D'autre part, la réécriture en partant de $\square \circ_{2}\left(\square \circ_{2} \square\right)$ donne

$$
\square \circ_{2}\left(\square \circ_{1} \square+\square \circ_{3} \square\right)=\left(\square \circ_{2} \square\right) \circ_{2} \square+\left(\square \circ_{2} \square\right) \circ_{4} \square \text {. }
$$


On utilise à nouveau (11) deux fois pour obtenir

$$
\begin{aligned}
\left(\square \circ_{1} \square+\square \circ_{3} \square\right) & \circ_{2} \square+\left(\square \circ_{1} \square+\square \circ_{3} \square\right) \circ_{4} \square= \\
& \square \circ_{1}\left(\square \circ_{2} \square\right)-\left(\square \circ_{2} \square\right) \circ_{5} \square-\left(\square \circ_{2} \square\right) \circ_{1} \square+\square \circ_{3}\left(\square \circ_{2} \square\right) .
\end{aligned}
$$

On utilise à nouveau (11) quatre fois pour obtenir

$$
\begin{aligned}
\square \circ_{1}\left(\square \circ_{1} \square+\square \circ_{3} \square\right)-\left(\square \circ_{1} \square+\square \circ_{3} \square\right) \circ_{5} \square \\
\quad-\left(\square \circ_{1} \square+\square \circ_{3} \square\right) \circ_{1} \square+\square \circ_{3}\left(\square \circ_{1} \square+\square \circ_{3} \square\right) .
\end{aligned}
$$

Dans cette somme, certains termes se simplifient par paires et il reste

$$
\square \circ_{1}\left(\square \circ_{3} \square\right)+\square \circ_{3}\left(\square \circ_{1} \square\right),
$$

ce qui est bien égal à l'autre réécriture.

Proposition 1.4 Pour tout $n \geq 0$, l'ensemble $\left(Q_{x}\right)_{x \in Y_{n}}$ forme une base de $\mathbf{V}(2 n+1)$.

Preuve. La propriété de Gröbner entraîne l'énoncé. On sait en effet qu'une base de l'opérade quotient par un idéal est donné par les monômes réduits relativement à une base de Gröbner de cet idéal. Dans le cas présent, les monômes réduits de l'opérade libre sur $\square$ par rapport à la relation (7), pour l'ordre admissible choisi, sont exactement les compositions itérées ne faisant pas intervenir la composition $\mathrm{o}_{2}$, i.e. (au signe près) les éléments de $Q$.

Corollaire 1.5 L'opérade $\mathbf{V}$ est de Koszul.

Preuve. Ceci résulte du fait général qu'une opérade ayant une base de Gröbner quadratique est de Koszul, voir par exemple [17, Th. 3.10].

\subsection{Produits associatifs sur V}

Par abus de notation, on note aussi $\mathbf{V}$ la somme directe

$$
\bigoplus_{n \geq 0} \mathbf{V}(2 n+1),
$$

qui est un groupe abélien doublement gradué par le degré et par le poids.

On introduit les notations alternatives suivantes :

$$
a / b=(-1)^{a b} b \circ_{1} a
$$

et

$$
a * b=a \circ_{\max } b .
$$

Proposition 1.6 Les produits / et $*$ sont deux produits associatifs sur $\mathbf{V}$, qui vérifient de plus la relation de compatibilité suivante:

$$
(a / b) * c=a /(b * c) .
$$




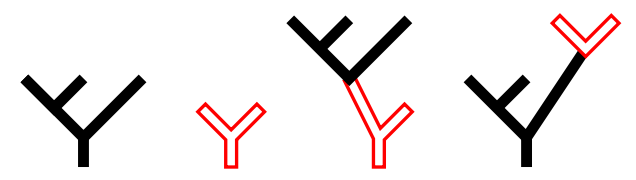

Figure 2 - Les opérations combinatoires / et $\backslash: x, y, x / y$ et $x \backslash y$

Preuve. L'associativité résulte de l'axiome (2) et la compatibilité de l'axiome (3) des opérades.

La définition récursive (9) de la base $Q$ se traduit, pour un arbre $z$ qui se décompose en une paire $(x, y)$ d'arbres, en fonction de / et * :

$$
Q_{z}=Q_{x} / \square * Q_{y}
$$

où l'on peut omettre les parenthèses par la proposition 1.6

On introduit deux opérations combinatoires sur les arbres binaires plans, voir figure 2 pour un exemple. Soient $x, y$ deux arbres binaires plans.

L'arbre binaire plan $x / y$ est obtenu par greffe à gauche de $x$ sur $y$, i.e. en identifiant l'arête incidente à la racine de $x$ avec l'arête incidente à la feuille la plus à gauche de $y$.

L'arbre binaire plan $x \backslash y$ est obtenu par greffe à droite de $y$ sur $x$, i.e. en identifiant l'arête incidente à la racine de $y$ avec l'arête incidente à la feuille la plus à droite de $x$.

Remarque 1.7 On a la relation $(x / y) \backslash z=x /(y \backslash z)$.

Lemme 1.8 On a la description suivante des opérations / et * dans la base $Q$ :

$$
\begin{aligned}
& Q_{x \backslash y}=Q_{x} * Q_{y}, \\
& Q_{x / y}=Q_{x} / Q_{y} .
\end{aligned}
$$

La famille $Q$ est donc close pour / et $*$.

Preuve. Ceci résulte facilement de la définition (16) de $Q_{x}$ et de la proposition 1.6. par récurrence.

\subsection{Caractérisation de $\theta_{\mathbf{V}}$}

Proposition 1.9 L'application $\theta_{\mathbf{V}}$ vérifie

$$
\theta_{\mathbf{V}}(a / b)=-\theta_{\mathbf{V}}(a) * \theta_{\mathbf{V}}(b) .
$$

Preuve. On utilise le second axiome des opérades anticycliques (6) et les définitions (13) et (14) des produits / et *.

Proposition 1.10 L'application $\theta_{\mathbf{V}}$ vérifie

$$
\theta_{\mathbf{V}}(\square *(a /(\square * b)))=\theta_{\mathbf{V}}(\square * a) / \theta_{\mathbf{V}}(\square * b)-\theta_{\mathbf{V}}(\square * a) * \theta_{\mathbf{V}}(\square * b) .
$$


Preuve. En revenant aux expressions en les compositions $\circ_{i}$, on calcule le terme de gauche en utilisant (5) :

$$
\begin{aligned}
(-1)^{a b+a} \theta_{\mathbf{V}}\left(\square \circ_{3}\left(\left(\square \circ_{3} b\right) \circ_{1} a\right)\right) & =-(-1)^{a b+a} \square \circ_{2}\left(\left(\square \circ_{3} b\right) \circ_{1} a\right) \\
& =-(-1)^{a b+a}\left(\left(\square \circ_{2} \square\right) \circ_{4} b\right) \circ_{2} a .
\end{aligned}
$$

En utilisant la relation (7) pour réécrire $\square \circ_{2} \square$, on obtient

$$
-(-1)^{a b+a}\left(\left(\square \circ_{1} \square\right) \circ_{4} b\right) \circ_{2} a-(-1)^{a b+a}\left(\left(\square \circ_{3} \square\right) \circ_{4} b\right) \circ_{2} a .
$$

Par application des axiomes d'opérades, ceci vaut

$$
(-1)^{a b+a+b+1}\left(\square \circ_{2} b\right) \circ_{1}\left(\square \circ_{2} a\right)-\left(\square \circ_{2} a\right) \circ_{\max }\left(\square \circ_{2} b\right) .
$$

En utilisant l'axiome (5) des opérades anticycliques, on obtient

$$
(-1)^{a b+a+b+1} \theta_{\mathbf{V}}\left(\square \circ_{3} b\right) \circ_{1} \theta_{\mathbf{V}}\left(\square \circ_{3} a\right)-\theta_{\mathbf{V}}\left(\square \circ_{3} a\right) \circ_{\max } \theta_{\mathbf{V}}\left(\square \circ_{3} b\right) .
$$

En repassant aux formules utilisant / et $*$, on trouve bien le second terme voulu.

Proposition 1.11 Les applications $\theta_{\mathbf{V}}$ sont uniquement déterminés par les conditions (17) et (18) et les conditions initiales

$$
\theta_{\mathbf{V}}(\mathbf{1})=-\mathbf{1} \quad \text { et } \quad \theta_{\mathbf{V}}(\square)=-\square .
$$

Preuve. Tout d'abord, la valeur de $\theta_{\mathbf{V}}\left(Q_{\mid}\right)$pour l'arbre trivial | est fixé par la première condition initiale, qui fait partie de la définition d'une opérade anticyclique.

On utilise ensuite le fait combinatoire élémentaire suivant. Soit $x$ un arbre non trivial. Alors ou bien $x$ peut s'écrire $y / z$ pour deux arbres non triviaux $y$ et $z$, ou bien $x$ peut s'écrire $Y \backslash z$ où $Y$ est l'unique arbre à un seul sommet et $z$ est un arbre éventuellement trivial.

Par conséquent, et par le lemme 1.8, tout élément $Q_{x}$ pour $x$ non trivial peut s'écrire soit comme $Q_{y} / Q_{z}$ soit comme $\square * Q_{z}$ avec les conditions précédemment décrites.

Si $Q_{x}$ s'écrit $Q_{y} / Q_{z}$, on peut définir $\theta_{\mathbf{V}}$ par récurrence par l'équation (17).

Sinon $Q_{x}$ s'écrit $\square * Q_{z}$. Si $z$ est trivial, on utilise la seconde condition initiale pour définir $\theta_{\mathbf{V}}\left(Q_{Y}\right)$. Sinon, on utilise la remarque combinatoire suivante : tout arbre $z$ non trivial peut s'écrire

$$
z=v /(\curlyvee \backslash w)
$$

où $Y$ est l'unique arbre à un sommet et $v, w$ sont deux arbres éventuellement triviaux. Par conséquent, on a

$$
Q_{z}=Q_{v} /\left(\square * Q_{w}\right) .
$$

On utilise alors (18) pour définir $\theta_{\mathbf{V}}\left(Q_{x}\right)$. 


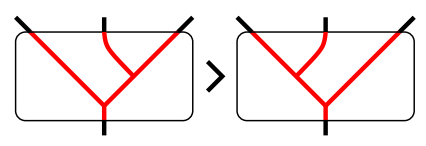

Figure 3 - Changement local : $y>x$

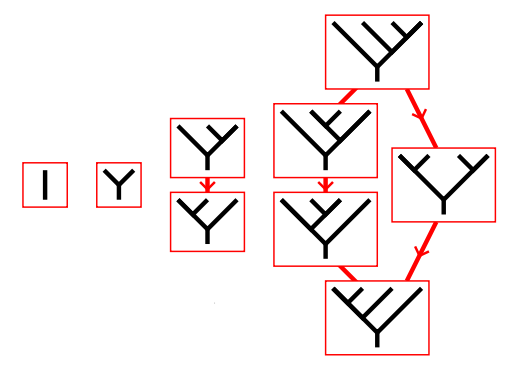

Figure 4 - Treillis de Tamari (minimum en bas)

\section{Posets de Tamari}

\subsection{Définition et modules}

Soit $n$ un entier positif ou nul et $\mathrm{Y}_{n}$ l'ensemble des arbres binaires plans à $n$ sommets internes. Dans 32, D. Tamari a défini un ordre partiel sur l'ensemble $\mathrm{Y}_{n}$ comme suit.

Un arbre $x$ est inférieur ou égal à un arbre $y(x \leq y)$ si on passe de $y$ à $x$ (dans cet ordre) par une suite de mouvements locaux qui changent une configuration "arête droite" en une configuration "arête gauche" selon le modèle de la figure 3

Friedman et Tamari [10] ont montré que ces posets sont des treillis. Une autre preuve a été donnée dans 18.

On renvoie le lecteur aux références [21, 5, 14, 22] pour la théorie des représentations des carquois, des posets et des algèbres de dimension finie.

On peut considérer le diagramme de Hasse de $\mathrm{Y}_{n}$ comme un carquois-avecrelations, en orientant les arêtes du maximum vers le minimum, et en imposant comme relations l'égalité de toute paire de chemins ayant mêmes débuts et fins.

On fixe désormais un corps de base $\mathrm{k}$ pour les modules sur les carquois. Les constructions qui suivent en dépendent peu et la dépendance en $\mathrm{k}$ sera implicite.

On considère alors la catégorie $\bmod Y_{n}$ des modules sur le carquois-avecrelations $\mathrm{Y}_{n}$. Cette catégorie est équivalente à la catégorie des modules sur l'algèbre d'incidence de $\mathrm{Y}_{n}$. C'est une catégorie abélienne de dimension globale finie.

On dispose de trois bases de l'anneau de Grothendieck $K_{0}\left(\bmod Y_{n}\right)$ données par les classes des modules projectifs, injectifs et simples sur $\mathrm{Y}_{n}$, notées respectivement $P_{x}, I_{x}$ et $S_{x}$ pour $x \in \mathrm{Y}_{n}$.

La base $S$ des simples est reliée à la base $P$ des projectifs par la formule

$$
P_{x}=\sum_{y \leq x} S_{y}
$$


et à la base $I$ des injectifs par la formule

$$
I_{x}=\sum_{y \geq x} S_{y} .
$$

\section{$2.2 \quad$ Structures algébriques}

On rappelle dans cette section des structures algébriques qui sont déjà bien étudiées, notamment dans les articles [16, 25, 1, 24]. Pour cette raison, on ne donne que des indications de preuves.

On considère maintenant la somme directe

$$
\bigoplus_{n \geq 0} K_{0}\left(\bmod Y_{n}\right)
$$

et plusieurs produits sur ce groupe abélien.

On a tout d'abord deux produits associatifs / et $\backslash$ définis sur la base $S$ par

$$
\begin{aligned}
& S_{x} / S_{y}=S_{x / y}, \\
& S_{x} \backslash S_{y}=S_{x \backslash y} .
\end{aligned}
$$

On a par ailleurs un autre produit associatif $*$, qui est le produit associatif de l'algèbre dendriforme libre. On peut le définir par la caractérisation suivante, due à Loday et Ronco [25].

Proposition 2.1 Soient $x, y$ deux arbres. Alors

$$
S_{x} * S_{y}=\sum_{x / y \leq z \leq x \backslash y} S_{z} .
$$

On peut par ailleurs donner du produit $*$ une description combinatoire alternative, voir [23, Prop. 5.11] pour un énoncé qui implique celui ci-dessous.

Proposition 2.2 Le produit $S_{x} * S_{y}$ est la somme des $S_{z}$ où $z$ décrit les arbres binaires plans obtenus en identifiant le coté droit de $x$ avec le coté gauche de $y$ par un homéomorphisme croissant qui n'envoie pas de sommet interne sur un sommet interne.

L'élément $S_{\mid}$est une unité pour les trois produits $/, \backslash$ et $*$.

Proposition 2.3 On a la description suivante des opérations / et $*$ dans la base P :

$$
\begin{aligned}
& P_{x \backslash y}=P_{x} * P_{y}, \\
& P_{x / y}=P_{x} / P_{y} .
\end{aligned}
$$

Preuve. Pour la première formule, il s'agit de voir que les arbres $z$ qui sont inférieurs ou égaux à l'arbre $x \backslash y$ (condition $\mathbf{A}$ ) sont exactement ceux qui s'obtiennent par recollement du bord droit d'un arbre inférieur à $x$ avec le bord gauche d'un arbre inférieur à $y$ (condition $\mathbf{B}$ ). Il est facile de déduire de la forme locale de l'ordre de Tamari que la condition $\mathbf{B}$ est stable par diminution dans le poset de Tamari, donc que la condition $\mathbf{A}$ entrâne la condition 
B. Réciproquement, étant donné un arbre $z$ vérifiant la condition $\mathbf{B}$, on peut l'écrire comme un des termes de $S_{x^{\prime}} * S_{y^{\prime}}$ avec $x^{\prime} \leq x$ et $y^{\prime} \leq y$. En appliquant une suite convenable de mouvements locaux définissant l'ordre de Tamari, on peut montrer que $z \leq x^{\prime} \backslash y^{\prime}$ Comme $x^{\prime} \backslash y^{\prime} \leq x \backslash y$, ceci entraîne que $z$ vérifie la condition A.

Montrons la seconde formule, en établissant l'énoncé suivant : pour tous $x, y$, on a une bijection

$$
\begin{aligned}
\left\{x^{\prime} \mid x^{\prime} \leq x\right\} \times\left\{y^{\prime} \mid y^{\prime} \leq y\right\} & \simeq\{z \mid z \leq x / y\} \\
\left(x^{\prime}, y^{\prime}\right) & \mapsto x^{\prime} / y^{\prime} .
\end{aligned}
$$

Le fait que $x^{\prime} / y^{\prime} \leq x / y$ résulte de la nature locale de l'ordre de Tamari. L'injectivité est claire. Pour montrer la surjectivité, il suffit de voir que l'existence pour $z$ d'une décomposition de la forme souhaitée entraîne l'existence d'une telle décomposition pour tout $z^{\prime}$ couvert par $z$. On conclut en partant de la décomposition de $x / y$.

En utilisant l'unique anti-automorphisme des treillis de Tamari, on obtient la proposition suivante.

Proposition 2.4 On a la description suivante des opérations $\backslash$ et $*$ dans la base I :

$$
\begin{aligned}
I_{x / y} & =I_{x} * I_{y}, \\
I_{x \backslash y} & =I_{x} \backslash I_{y} .
\end{aligned}
$$

On note $\theta$ la transformation de Coxeter du poset $Y_{n}$. C'est un endomorphisme de $K_{0}\left(\bmod Y_{n}\right)$ qui provient d'un endofoncteur $\tau_{A R}$ de la catégorie dérivée de $\bmod Y_{n}$.

L'endomorphisme $\theta$ est défini sur $K_{0}\left(\bmod Y_{n}\right)$ par la formule

$$
\theta\left(P_{x}\right)=-I_{x}
$$

pour tout $x \in \mathrm{Y}_{n}$.

Proposition 2.5 Les applications $\theta$ vérifient

$$
\begin{aligned}
\theta\left(P_{Y}\right) & =-P_{Y}, \\
\theta(a / b) & =-\theta(a) * \theta(b), \\
\theta(a * b) & =-\theta(a) \backslash \theta(b) .
\end{aligned}
$$

Preuve. La première formule pour $P_{Y}$ est immédiate, car $P_{Y}=I_{Y}$.

Pour la seconde formule, on calcule

$$
\theta\left(P_{x} / P_{y}\right)=\theta\left(P_{x / y}\right)=-I_{x / y}=-I_{x} * I_{y}=-\theta\left(P_{x}\right) * \theta\left(P_{y}\right) .
$$

Pour la troisième formule, on calcule

$$
\theta\left(P_{x} * P_{y}\right)=\theta\left(P_{x \backslash y}\right)=-I_{x \backslash y}=-I_{x} \backslash I_{y}=-\theta\left(P_{x}\right) \backslash \theta\left(P_{y}\right) .
$$


Lemme 2.6 On a

$$
\left(P_{Y} \backslash a\right) *\left(P_{Y} \backslash b\right)=P_{Y} \backslash\left(a *\left(P_{Y} \backslash b\right)\right)+\left(P_{Y} \backslash a\right) /\left(P_{Y} \backslash b\right) .
$$

Preuve. Ceci résulte de la description des trois produits /, \et $*$ dans la base $S$, voir (25), (26) et la proposition 2.2. Le produit $\left(P_{Y} \backslash a\right) *\left(P_{Y} \backslash b\right)$ se coupe naturellement en deux termes selon que le sommet interne inférieur provienne de $P_{Y} \backslash a$ ou de $P_{Y} \backslash b$.

\section{Proposition 2.7 On a}

$$
\theta\left(P_{Y} *\left(a /\left(P_{Y} * b\right)\right)\right)=\theta\left(P_{\curlyvee} * a\right) / \theta\left(P_{\curlyvee} * b\right)-\theta\left(P_{Y} * a\right) * \theta\left(P_{Y} * b\right) .
$$

Preuve. On calcule le terme de gauche :

$$
-\theta\left(P_{Y}\right) \backslash \theta\left(a /\left(P_{Y} * b\right)\right)=-\theta\left(P_{Y}\right) \backslash\left(\theta(a) *\left(\theta\left(P_{Y}\right) \backslash \theta(b)\right)\right),
$$

soit

$$
-P_{Y} \backslash\left(\theta(a) *\left(P_{Y} \backslash \theta(b)\right)\right) .
$$

On calcule le terme de droite :

$$
\left(\theta\left(P_{Y}\right) \backslash \theta(a)\right) /\left(\theta\left(P_{Y}\right) \backslash \theta(b)\right)-\left(\theta\left(P_{Y}\right) \backslash \theta(a)\right) *\left(\theta\left(P_{Y}\right) \backslash \theta(b)\right)
$$

soit

$$
\left(P_{Y} \backslash \theta(a)\right) /\left(P_{Y} \backslash \theta(b)\right)-\left(P_{Y} \backslash \theta(a)\right) *\left(P_{Y} \backslash \theta(b)\right) .
$$

Par le lemme 2.6. on déduit l'égalité voulue.

\section{Isomorphisme}

Pour tout $n \geq 0$, on définit une application linéaire $\psi$ de $\mathbf{V}(2 n+1)$ dans $K_{0}\left(\bmod Y_{n}\right)$ par

$$
\psi\left(Q_{x}\right)=P_{x},
$$

pour tout arbre binaire plan $x$.

Proposition 3.1 L'application $\psi$ est un isomorphisme de groupes abéliens.

Preuve. En effet, l'ensemble $\left(P_{x}\right)_{x \in \mathrm{Y}_{n}}$ est une base de $K_{0}\left(\bmod \mathrm{Y}_{n}\right)$.

Proposition 3.2 L'application $\psi$ est un morphisme pour / et $*$.

Preuve. Ceci résulte de la description identique de ces produits dans les bases $Q$ et $P$ à l'aide des opérations combinatoires / et $\backslash$ sur les arbres binaires plans, par les propositions 1.8 et 2.3 .

Théorème 3.3 L'isomorphisme $\psi$ de $\mathbf{V}(2 n+1)$ dans $K_{0}\left(\bmod \mathrm{Y}_{n}\right)$ transforme l'endomorphisme $\theta_{\mathbf{V}}$ en l'endomorphisme $\theta$ :

$$
\psi \theta_{\mathbf{V}}=\theta \psi
$$


Preuve. L'idée de la preuve est la suivante : les morphismes $\theta_{\mathbf{V}}$ et $\theta$ sont caractérisés par des propriétés similaires qui ne font intervenir que les produits * et $/$.

Pour faciliter la preuve, on identifie $P_{x}$ avec $Q_{x}$ pour tout $x$. On va donc montrer que $\theta_{\mathbf{v}}=\theta$.

Vérifions d'abord les conditions initiales. Pour $n=0, \theta_{\mathbf{V}}=-$ Id car $\mathbf{V}$ est une opérade anticyclique et $\theta=-\mathrm{Id}$. Pour $n=1, \theta_{\mathbf{V}}=-\mathrm{Id}$ et $\theta=-\mathrm{Id}$.

En utilisant les propositions 2.5 et 2.7 on vérifie que la collection de morphismes $\theta$ satisfait, après identification des bases $Q$ et $P$, les conditions (17) et (18) qui caractérisent $\theta_{\mathbf{V}}$ selon la proposition 1.11 .

\section{Application au polynôme caractéristique}

On va utiliser le théorème 3.3 et la théorie de la dualité de Koszul des opérades pour calculer le polynôme caractéristique de la transformation de Coxeter $\theta$ du poset de Tamari $Y_{n}$.

\subsection{Structure cyclique et dualité de Koszul}

Par commodité, on va plutôt utiliser une structure cyclique sur $\mathbf{V}$, qui se trouve être donnée par l'opposé de la structure anticyclique.

Proposition 4.1 On peut munir $\mathbf{V}$ d'une structure cyclique définie par $\gamma_{\mathbf{V}}(\square)=$ $\square$. On a alors la relation globale $\gamma_{\mathbf{V}}=-\theta_{\mathbf{V}}$.

Preuve. La vérification de la compatibilité de $\gamma_{\mathbf{V}}$ avec la relation (7) est immédiate. En effet, l'image par $\gamma_{\mathbf{V}}$ de cette relation est

$$
-\square \circ_{3} \square-\square \circ_{1} \square+\square \circ_{2} \square .
$$

Pour le reste, il suffit de voir que $-\gamma_{\mathbf{V}}$ définit une structure anticyclique qui coïncide avec $\theta_{\mathbf{V}}$ sur $\square$. Les deux axiomes d'opérade cyclique pour $\gamma_{\mathbf{V}}$ impliquent les deux axiomes d'opérade anticyclique pour $-\gamma_{\mathbf{V}}$. De plus, $-\gamma_{\mathbf{V}}$ est bien d'ordre $2 n+2$ sur l'espace $\mathbf{V}(2 n+1)$ pour tout $n$.

Décrivons l'opérade $\mathbf{W}$ duale de $\mathbf{V}$ (voir 27] pour la procédure de calcul de l'opérade duale). Elle est engendrée par un élément $w$ de poids 0 et de degré 3 vérifiant les relations suivantes :

$$
w \circ_{1} w+w \circ_{2} w=0 \quad \text { et } \quad w \circ_{2} w+w \circ_{3} w=0 .
$$

Par dualité de Koszul, comme V est de Koszul, W l'est aussi. On a la description suivante :

Proposition 4.2 L'opérade $\mathbf{W}$ est de dimension 1 en chaque degré impair et une base de $\mathbf{W}(2 n+1)$ est donnée par $w_{n}=w \circ_{1} \cdots \circ_{1} w$ avec $n$ copies de $w$.

Proposition 4.3 On peut munir $\mathbf{W}$ d'une structure cyclique définie par $\gamma(w)=$ $-w$. On a alors $\gamma\left(w_{n}\right)=(-1)^{n} w_{n}$. 
Preuve. Pour l'existence, il suffit de vérifier la compatibilité de $\gamma$ avec les relations de l'opérade $\mathbf{W}$. Pour la valeur de $\gamma$ sur $w_{n}$, il suffit de faire une récurrence sur $n$. En effet, on a

$$
\gamma\left(w_{n}\right)=\gamma\left(w_{n-1} \circ_{1} w\right)=-w \circ_{3} \gamma\left(w_{n-1}\right)=(-1)^{n} w \circ_{3} w_{n-1} .
$$

On montre par ailleurs par récurrence sur $n$ que $w \circ_{3} w_{n-1}=w_{n}$, en utilisant la relation $w \circ_{1} w=w \circ_{3} w$.

Remarque 4.4 On peut vérifier que cette structure cyclique sur $\mathbf{W}$ est celle qui provient, via le quasi-isomorphisme entre $\mathrm{BV}$ et $\mathbf{W}$, de la structure cyclique naturelle sur la cobar-construction BV.

\subsection{Rappels sur les fonctions symétriques}

On utilise les notations standards pour les fonctions symétriques, qui sont celles du livre [26.

Soit $\Lambda$ l'anneau des fonctions symétriques sur $\mathbb{Q}$. On note $\left(p_{n}\right)_{n \geq 1}$ les fonctions symétriques "sommes de puissances". L'anneau $\Lambda$ est l'anneau des polynômes en les $\left(p_{n}\right)_{n \geq 1}$. Il admet une base $\left(p_{\lambda}\right)_{\lambda}$, formée des monômes en les $\left(p_{n}\right)_{n \geq 1}$ et indexée par les partitions d'entiers. On munit $\Lambda$ d'une graduation naturelle en posant $\operatorname{deg}\left(p_{i}\right)=i$. On travaille par la suite dans le complété de l'anneau $\Lambda$ par rapport à sa graduation.

On note $\Sigma$ la suspension des fonctions symétriques, définie par

$$
(\Sigma f)\left(p_{1}, p_{2}, \ldots, p_{i}, \ldots\right)=-f\left(-p_{1},-p_{2}, \ldots,-p_{i}, \ldots\right) .
$$

On note $\omega$ l'automorphisme involutif des fonctions symétriques qui est défini par

$$
(\omega f)\left(p_{1}, p_{2}, \ldots, p_{i}, \ldots\right)=f\left(-p_{1}, p_{2}, \ldots,(-1)^{i-1} p_{i}, \ldots\right) .
$$

Si $\rho$ est un caractère du groupe symétrique $\mathfrak{S}_{n}$, on identifiera $\rho$ à la fonction symétrique

$$
\sum_{\lambda \vdash n} \rho\left(C_{\lambda}\right) \frac{p_{\lambda}}{z_{\lambda}}
$$

où la somme porte sur les partitions de $n, C_{\lambda}$ est la classe de conjugaison de type cyclique $\lambda$ et $z_{\lambda}\left|C_{\lambda}\right|=n$ !.

On note $\circ$ le pléthysme des fonctions symétriques, pour lequel on renvoie à la littérature.

On appelle série caractéristique d'une opérade cyclique $\mathbf{P}$ la fonction symétrique

$$
\mathrm{Ch}_{\mathbf{P}}=\sum_{n \geq 1} \operatorname{Ind}_{\mathbb{Z} / n+1}^{\mathfrak{S}_{n+1}}\left(\sum_{k \in \mathbb{Z}}(-1)^{k} \chi_{\mathbf{P}(n)_{k}}\right)
$$

où $\chi_{\mathbf{P}(n)_{k}}$ est le caractère de $\mathbb{Z}_{/ n+1}$ sur $\mathbf{P}(n)_{k}$, la composante de poids $k$ de $\mathbf{P}(n)$.

On rappelle maintenant la transformation de Legendre des fonctions symétriques, introduite par Getzler et Kapranov dans [13, §7].

Soient $A$ et $B$ deux fonctions symétriques sans termes de degré inférieur ou égal à 1 et tels que les termes de degré 1 de $\partial_{p_{1}} A$ et de $\partial_{p_{1}} B$ sont non nuls. On dit que $A$ est la transformée de Legendre de $B$ si

$$
A \circ \partial_{p_{1}} B+B=p_{1} \partial_{p_{1}} B .
$$


La transformation de Legendre est une involution. Dans cette situation, on a aussi une relation entre les dérivées partielles de $A$ et de $B$ par rapport à $p_{1}$ :

$$
\partial_{p_{1}} A \circ \partial_{p_{1}} B=p_{1} .
$$

La dualité de Koszul est reliée comme suit à la transformée de Legendre, voir [13, §7] et en particulier le corollaire (7.22).

Proposition 4.5 Lorsque deux opérades cycliques $\mathbf{V}$ et $\mathbf{W}$ sont de Koszul et duales l'une de l'autre (au sens cyclique) alors la série caractéristique $\mathrm{Ch}_{\mathbf{V}}$ est la transformée de Legendre de $-\Sigma \mathrm{Ch}_{\mathbf{w}}$.

Dans cet énoncé, "au sens cyclique" signifie que la structure cyclique de $\mathbf{W}$ provient de celle de $\mathbf{V}$ via la cobar-construction $\mathbf{B V}$ vue comme opérade cyclique et le quasi-isomorphisme entre BV et $\mathbf{W}$. Dans ce cas, on a une égalité

$$
\mathrm{Ch}_{\mathbf{W}}=\mathrm{Ch}_{\mathrm{BV}} \cdot
$$

Pour plus de détails sur le cadre théorique de la proposition 4.5, le lecteur pourra consulter [13, 12, 11.

\subsection{Séries caractéristiques de $\mathrm{V}$ et $\mathrm{W}$}

Proposition 4.6 La série caractéristique de l'opérade cyclique $\mathbf{W}$ est

$$
\mathrm{Ch}_{\mathbf{W}}=\sum_{n \geq 1} \frac{1}{2 n} \sum_{j \mid 2 n}(-1)^{j(n-1)} \phi(2 n / j) p_{2 n / j}^{j},
$$

où $\phi$ est l'indicatrice d'Euler.

Preuve. Par la définition de $\mathrm{Ch}_{\mathbf{W}}$ et par la proposition 4.3, on a

$$
\mathrm{Ch}_{\mathbf{W}}=\sum_{n \geq 1} \operatorname{Ind}_{\mathbb{Z} / 2 n}^{\mathfrak{S}_{2 n}}(-1)^{n-1}
$$

où $(-1)^{n-1}$ est la caractère valant $(-1)^{n-1}$ sur le générateur de $\mathbb{Z}_{/ 2 n}$. Par un calcul standard du caractère induit, on trouve que

$$
\mathrm{Ch}_{\mathbf{W}}=\sum_{n \geq 1} \frac{1}{2 n} \sum_{j \mid 2 n} \sum_{\substack{i=1 \\ i \wedge 2 n / j=1}}^{2 n / j}(-1)^{i j(n-1)} p_{2 n / j}^{j} .
$$

Il suffit enfin de remarquer (en distinguant le cas où $j$ est impair et $n$ pair) que

$$
\sum_{\substack{i=1 \\ i \wedge 2 n / j=1}}^{2 n / j}(-1)^{i j(n-1)}=(-1)^{j(n-1)} \phi(2 n / j) .
$$

Par la remarque 4.4, la proposition 4.5 et la proposition 4.12, on a donc 
Proposition 4.7 La série caractéristique de l'opérade cyclique $\mathbf{V}$ est

$$
\mathrm{Ch}_{\mathbf{V}}=\sum_{n \geq 1}(-1)^{n-1} c_{n-1} p_{1}^{2 n}+\sum_{n \geq 1} \frac{1}{2 n} \sum_{j \mid 2 n} \lambda(2 n / j) \phi(j)(-1)^{2 n(n-1) / j} p_{j}^{2 n / j},
$$

où

$$
\lambda(n)=(-1)^{\left(\begin{array}{c}
n \\
2
\end{array}\right)}\left(\begin{array}{c}
n-1 \\
\left\lfloor\frac{n-1}{2}\right\rfloor
\end{array}\right) \quad \text { et } \quad c_{n}=\frac{1}{n+1}\left(\begin{array}{c}
2 n \\
n
\end{array}\right) .
$$

\subsection{Polynôme caractéristique de $\theta_{\mathrm{V}}$}

On introduit la suite $\left(b_{n}\right)_{n \geq 1}$ définie pour tout $n \geq 1$ par

$$
b_{n}=\frac{1}{n} \sum_{d \mid n} \mu(d) \lambda(n / d),
$$

où $\mu$ est la fonction de Möbius et $\lambda$ est définie dans (50). On peut montrer que les $b_{n}$ sont des entiers relatifs, voir [3, §3]. Par inversion de Möbius, on a

$$
\sum_{d \mid n} d b_{d}=\lambda(n)
$$

Soit $n$ un entier et $d$ divisant $n$. On note $M_{n, d}$ le module $\mathbb{Q}[t] /\left(t^{d}-1\right)$ sur lequel le générateur de $\mathbb{Z}_{/ n}$ agit par multiplication par $t$. Soit $M_{n, d}^{\prime}$ le module induit de $\mathbb{Z}_{/ n}$ à $\mathfrak{S}_{n}$ de $M_{n, d}$. Le caractère de $M_{n, d}^{\prime}$ est donné (voir [3, §1.4]) par la formule :

$$
\frac{d}{n} \sum_{\ell \mid n / d} \phi(\ell) p_{\ell}^{n / \ell}
$$

En particulier, le caractère de $M_{n, n}^{\prime}$ est $p_{1}^{n}$.

La proposition 4.7 se traduit en l'assertion suivante.

Proposition 4.8 L'action du groupe cyclique $\mathbb{Z}_{/ 2 n+2}$ engendré par $(-1)^{n+1} \theta_{\mathbf{V}}$ sur l'espace vectoriel $\mathbf{V}(2 n+1)$ est donnée par le module virtuel

$$
c_{n} M_{2 n+2,2 n+2}-(-1)^{n+1} \sum_{d \mid 2 n+2} b_{d} M_{2 n+2, d} .
$$

Preuve. La formule pour $\mathrm{Ch}_{\mathbf{V}}$ de la prop. 4.7 peut s'écrire, en utilisant (52), comme

$$
\sum_{n \geq 1}(-1)^{n-1} c_{n-1} p_{1}^{2 n}+\sum_{n \geq 1} \frac{1}{2 n} \sum_{j \mid 2 n} j b_{j} \sum_{\ell \mid 2 n / j} \phi(\ell)(-1)^{2 n(n-1) / \ell} p_{\ell}^{2 n / \ell} .
$$

En tenant compte du fait que $\mathbf{V}(2 n-1)$ est en poids $n-1$, on obtient pour l'action de $\gamma_{\mathbf{V}}=-\theta_{\mathbf{V}}$, la formule

$$
\sum_{n \geq 1} c_{n-1} p_{1}^{2 n}+(-1)^{n-1} \sum_{n \geq 1} \sum_{j \mid 2 n} b_{j} \frac{j}{2 n} \sum_{\ell \mid 2 n / j} \phi(\ell)(-1)^{2 n(n-1) / \ell} p_{\ell}^{2 n / \ell} .
$$

En faisant agir $\omega^{n-1}$ sur la partie de degré $2 n$, on obtient

$$
\sum_{n \geq 1} c_{n-1} p_{1}^{2 n}-(-1)^{n} \sum_{n \geq 1} \sum_{j \mid 2 n} b_{j} \frac{j}{2 n} \sum_{\ell \mid 2 n / j} \phi(\ell) p_{\ell}^{2 n / \ell}
$$


qui décrit les actions de $(-1)^{n} \theta \mathbf{V}$ sur $\mathbf{V}(2 n-1)$, par le lemme 4.10

Enfin, on reconnaît la formule (53), et on obtient l'expression virtuelle

$$
c_{n} M_{2 n+2,2 n+2}^{\prime}-(-1)^{n+1} \sum_{d \mid 2 n+2} b_{d} M_{2 n+2, d}^{\prime}
$$

pour la représentation induite de $(-1)^{n+1} \theta_{\mathbf{V}}$ sur $\mathbf{V}(2 n+1)$. On en déduit l'énoncé en utilisant l'injectivité de l'application linéaire Ind (voir [ㅍ, §1.4]).

Remarque 4.9 La forme de cette proposition fait penser à l'existence possible d'une suite exacte courte. Il serait intéressant de décrire explicitement une telle suite exacte.

Lemme 4.10 On a un carré commutatif

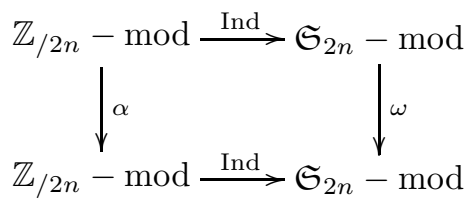

où $\alpha$ est l'application qui multiplie par -1 l'action du générateur de $\mathbb{Z}_{/ 2 n}$ et $\omega$ est l'involution des fonctions symétriques définie par (42).

Preuve. La formule pour l'induite d'un caractère $\chi$ est donnée par

$$
\frac{1}{2 n} \sum_{j \mid 2 n} \sum_{\substack{i=1 \\ i \wedge 2 n / j=1}}^{2 n / j} \chi(j i) p_{2 n / j}^{j} .
$$

Pour le caractère $\alpha(\chi)$ tordu par le signe, on obtient donc

$$
\frac{1}{2 n} \sum_{j \mid 2 n} \sum_{\substack{i=1 \\ i \wedge 2 n / j=1}}^{2 n / j}(-1)^{j i} \chi(j i) p_{2 n / j}^{j} .
$$

Mais dans cette somme, $(-1)^{j i}$ vaut toujours $(-1)^{j}$, que $j$ soit pair ou impair. On obtient ainsi la formule pour l'image par $\omega$ de l'induite de $\chi$, et donc le carré commutatif voulu.

On déduit de la proposition 4.8 l'énoncé suivant.

Théorème 4.11 Le polynôme caractéristique de la transformation de Coxeter du poset de Tamari $\mathrm{Y}_{n}$ est donné par

$$
\frac{\left(x^{2 n+2}-1\right)^{c_{n}}}{\left(\prod_{d \mid 2 n+2}\left(x^{d}-(-1)^{d(n+1)}\right)^{b_{d}}\right)^{(-1)^{n+1}}} .
$$


Preuve. Comme le polynôme caractéristique de $M_{n, d}$ est $x^{d}-1$, on déduit de la proposition 4.8 que le polynôme caractéristique de $(-1)^{n+1} \theta_{\mathbf{V}}$ sur $\mathbf{V}(2 n+1)$ est

$$
\frac{\left(x^{2 n+2}-1\right)^{c_{n}}}{\left(\prod_{d \mid 2 n+2}\left(x^{d}-1\right)^{b_{d}}\right)^{(-1)^{n+1}}} .
$$

Pour obtenir le polynôme caractéristique de $\theta_{\mathbf{V}}$ (qui est aussi celui de $\theta$ par le théorème 3.3), il suffit de remplacer $x$ par $(-1)^{n+1} x$ et de simplifier les signes.

Ceci démontre les conjectures 3.4 et 3.5 de $\underline{3}$, modulo une reformulation simple.

\subsection{Calcul de la transformée de Legendre}

Cette section est consacrée au calcul de la transformée de Legendre de la fonction symétrique associée à l'opérade cyclique $\mathbf{W}$.

On introduit la fonction symétrique $A$ (qui correspond à $\mathrm{Ch}_{\mathbf{V}}$ ) :

$$
A=\sum_{n \geq 1}(-1)^{n-1} c_{n-1} p_{1}^{2 n}+\sum_{n \geq 1} \frac{1}{2 n} \sum_{j \mid 2 n} \lambda(2 n / j) \phi(j)(-1)^{2 n(n-1) / j} p_{j}^{2 n / j},
$$

où $\lambda$ et $c_{n}$ sont définis dans (50). On considère aussi la fonction symétrique $B$ (qui correspond à $-\Sigma \mathrm{Ch}_{\mathbf{W}}$ ) :

$$
B=\sum_{n \geq 1} \frac{1}{2 n} \sum_{j \mid 2 n}(-1)^{2 n n / j} \phi(j) p_{j}^{2 n / j} .
$$

On calcule sans difficulté les dérivées partielles par rapport à $p_{1}$ :

$$
\partial_{p_{1}} A=\sum_{n \geq 1}(-1)^{n-1} c_{n-1} p_{1}^{2 n-1}
$$

et

$$
\partial_{p_{1}} B=\frac{p_{1}}{1-p_{1}^{2}} .
$$

On remarque que le premier terme de $A$ est $p_{1} \partial_{p_{1}} A$.

Proposition 4.12 La fonction A est la transformée de Legendre de B.

Preuve. Calculons d'abord $p_{1} \partial_{p_{1}} B-B$ :

$$
\frac{p_{1}^{2}}{1-p_{1}^{2}}-\sum_{n \geq 1} \frac{1}{2 n} \sum_{j \mid 2 n}(-1)^{2 n n / j} \phi(j) p_{j}^{2 n / j} .
$$

D'autre part, $A \circ \partial_{p_{1}} B$ vaut

$$
\left(\partial_{p_{1}} B\right)\left(\partial_{p_{1}} A \circ \partial_{p_{1}} B\right)+\sum_{n \geq 1} \frac{1}{2 n} \sum_{j \mid 2 n} \lambda(2 n / j) \phi(j)(-1)^{2 n(n-1) / j}\left(\frac{p_{j}}{1-p_{j}^{2}}\right)^{2 n / j},
$$


ce qui donne

$$
\frac{p_{1}^{2}}{1-p_{1}^{2}}+\sum_{n \geq 1} \frac{1}{2 n} \sum_{j \mid 2 n} \lambda(2 n / j) \phi(j)(-1)^{2 n(n-1) / j}\left(\frac{p_{j}}{1-p_{j}^{2}}\right)^{2 n / j} .
$$

Il suffit donc de montrer l'égalité entre les seconds termes. En échangeant les sommations, on obtient d'une part

$$
-\sum_{j \geq 1} \frac{\phi(j)}{j} \sum_{\substack{n \geq 1 \\ j \mid 2 n}} \frac{j}{2 n}(-1)^{2 n n / j} p_{j}^{2 n / j}
$$

et d'autre part

$$
\sum_{j \geq 1} \frac{\phi(j)}{j} \sum_{\substack{n \geq 1 \\ j \mid 2 n}} \frac{j}{2 n} \lambda(2 n / j)(-1)^{2 n(n-1) / j}\left(\frac{p_{j}}{1-p_{j}^{2}}\right)^{2 n / j} .
$$

Leur égalité résulte du lemme 4.13 .

Lemme 4.13 Pour tout $j \geq 1$ fixé, on a

$$
-\sum_{\substack{n \geq 1 \\ j \mid 2 n}} \frac{j}{2 n}(-1)^{2 n n / j} x^{2 n / j}=\sum_{\substack{n \geq 1 \\ j \mid 2 n}} \frac{j}{2 n} \lambda(2 n / j)(-1)^{2 n(n-1) / j}\left(\frac{x}{1-x^{2}}\right)^{2 n / j} .
$$

Preuve. On peut réécrire cette égalité comme suit :

$$
-\sum_{\substack{N \geq 1 \\ j N \text { pair }}} \frac{1}{N}(-1)^{j N^{2} / 2} x^{N}=\sum_{\substack{N \geq 1 \\ j N \text { pair }}} \frac{1}{N} \lambda(N)(-1)^{j N / 2+N}\left(\frac{x}{1-x^{2}}\right)^{N} .
$$

Pour vérifier cette relation, on distingue le cas $j$ pair du cas $j$ impair.

Si $j$ est pair, on doit vérifier que

$$
-\sum_{N \geq 1} \frac{1}{N}(-1)^{j N / 2} x^{N}=\sum_{N \geq 1} \frac{1}{N} \lambda(N)(-1)^{j N / 2+N}\left(\frac{x}{1-x^{2}}\right)^{N} .
$$

Ceci résulte aisément du développement de Taylor

$$
\sum_{N \geq 1} \frac{1}{N} \lambda(N) y^{N}=-\log \left(\frac{1-2 y+\sqrt{1+4 y^{2}}}{2}\right) .
$$

Si $j$ est impair, on doit vérifier que

$$
-\sum_{N \geq 1} \frac{1}{2 N} x^{2 N}=\sum_{N \geq 1} \frac{1}{2 N} \lambda(2 N)\left(\frac{x}{1-x^{2}}\right)^{2 N} .
$$

Ceci résulte du développement de Taylor

$$
\sum_{N \geq 1} \frac{1}{2 N}\left(\begin{array}{c}
2 N \\
N
\end{array}\right) y^{N}=-\log \left(\frac{1+\sqrt{1-4 y^{2}}}{2}\right) .
$$

Pour ces deux développements de Taylor, le lecteur peut consulter [3, Append. A]. 


\section{Références}

[1] Marcelo Aguiar and Frank Sottile. Structure of the Loday-Ronco Hopf algebra of trees. J. Algebra, 295(2) :473-511, 2006.

[2] Frédéric Chapoton. On the Coxeter transformations for Tamari posets. Canad. Math. Bull., 50(2) :182-190, 2007.

[3] Frédéric Chapoton. Le module dendriforme sur le groupe cyclique. Ann. Inst. Fourier (Grenoble), 58(7) :2333-2350, 2008.

[4] Frédéric Chapoton. Categorification of the dendriform operad. In JeanLouis Loday and Bruno Vallette, editors, Proceedings of Operads 2009, Séminaire et Congrès. SMF, 2012. oai :arXiv.org :0909.2751.

[5] Charles W. Curtis and Irving Reiner. Representation theory of finite groups and associative algebras. Pure and Applied Mathematics, Vol. XI. Interscience Publishers, a division of John Wiley \& Sons, New York-London, 1962.

[6] Vladimir Dotsenko and Anton Khoroshkin. Gröbner bases for operads. Duke Math. J., 153(2) :363-396, 2010.

[7] Kurusch Ebrahimi-Fard and Dominique Manchon. Dendriform equations. J. Algebra, 322(11) :4053-4079, 2009.

[8] Kurusch Ebrahimi-Fard, Dominique Manchon, and Frédéric Patras. New identities in dendriform algebras. J. Algebra, 320(2) :708-727, 2008.

[9] Sergey Fomin and Andrei Zelevinsky. Cluster algebras. I. Foundations. J. Amer. Math. Soc., 15(2) :497-529 (electronic), 2002.

[10] Haya Friedman and Dov Tamari. Problèmes d'associativité : Une structure de treillis finis induite par une loi demi-associative. J. Combinatorial Theory, 2 :215-242, 1967.

[11] E. Getzler. Operads and moduli spaces of genus 0 Riemann surfaces. In The moduli space of curves (Texel Island, 1994), volume 129 of Progr. Math., pages 199-230. Birkhäuser Boston, Boston, MA, 1995.

[12] E. Getzler and M. M. Kapranov. Cyclic operads and cyclic homology. In Geometry, topology, $\&$ physics, Conf. Proc. Lecture Notes Geom. Topology, IV, pages 167-201. Int. Press, Cambridge, MA, 1995.

[13] E. Getzler and M. M. Kapranov. Modular operads. Compositio Math., 110(1) :65-126, 1998.

[14] Dieter Happel. Triangulated categories in the representation theory of finite-dimensional algebras, volume 119 of London Mathematical Society Lecture Note Series. Cambridge University Press, Cambridge, 1988.

[15] Dieter Happel and Luise Unger. On a partial order of tilting modules. Algebr. Represent. Theory, 8(2) :147-156, 2005.

[16] F. Hivert, J.-C. Novelli, and J.-Y. Thibon. The algebra of binary search trees. Theoret. Comput. Sci., 339(1) :129-165, 2005.

[17] Eric Hoffbeck. A Poincaré-Birkhoff-Witt criterion for Koszul operads. $M a$ nuscripta Math., 131(1-2) :87-110, 2010.

[18] Samuel Huang and Dov Tamari. Problems of associativity : A simple proof for the lattice property of systems ordered by a semi-associative law. $J$. Combinatorial Theory Ser. A, 13 :7-13, 1972. 
[19] Sefi Ladkani. Universal derived equivalences of posets of cluster tilting objects, 2007.

[20] Sefi Ladkani. Universal derived equivalences of posets of tilting modules, 2007.

[21] Sefi Ladkani. On derived equivalences of categories of sheaves over finite posets. J. Pure Appl. Algebra, 212(2) :435-451, 2008.

[22] Helmut Lenzing. Coxeter transformations associated with finitedimensional algebras. In Computational methods for representations of groups and algebras (Essen, 1997), volume 173 of Progr. Math., pages 287308. Birkhäuser, Basel, 1999.

[23] Jean-Louis Loday. Dialgebras. In Dialgebras and related operads, volume 1763 of Lecture Notes in Math., pages 7-66. Springer, Berlin, 2001.

[24] Jean-Louis Loday. Arithmetree. J. Algebra, 258(1) :275-309, 2002. Special issue in celebration of Claudio Procesi's 60th birthday.

[25] Jean-Louis Loday and María O. Ronco. Order structure on the algebra of permutations and of planar binary trees. J. Algebraic Combin., 15(3) :253$270,2002$.

[26] I. G. Macdonald. Symmetric functions and Hall polynomials. Oxford Mathematical Monographs. The Clarendon Press Oxford University Press, New York, second edition, 1995. With contributions by A. Zelevinsky, Oxford Science Publications.

[27] Martin Markl and Elisabeth Remm. (non-)koszulity of operads for n-ary algebras, cohomology and deformations, 2009.

[28] Martin Markl, Steve Shnider, and Jim Stasheff. Operads in algebra, topology and physics, volume 96 of Mathematical Surveys and Monographs. American Mathematical Society, Providence, RI, 2002.

[29] Nathan Reading. Cambrian lattices. Adv. Math., 205(2) :313-353, 2006.

[30] Christine Riedtmann and Aidan Schofield. On a simplicial complex associated with tilting modules. Comment. Math. Helv., 66(1):70-78, 1991.

[31] María Ronco. Primitive elements in a free dendriform algebra. In New trends in Hopf algebra theory (La Falda, 1999), volume 267 of Contemp. Math., pages 245-263. Amer. Math. Soc., Providence, RI, 2000.

[32] Dov Tamari. The algebra of bracketings and their enumeration. Nieuw Arch. Wisk. (3), $10: 131-146,1962$.

[33] Bruno Vallette and Jean-Louis Loday. Algebraic Operads. a paraitre, 2010. xviii+512 pp. 